\title{
Supplementary Material \\ Modularity in protein Structures: study on all-alpha proteins
}

\author{
December 6, 2014
}

\section{Datasets:}

Four datasets have been used in the study to compare the distribution of patterns as well as to check the robustness of methodology. Maintaining the structural quality, datasets has been generated to establish the consistency in results with varying sequence identity as well as versions. Figure 1, illustrates the population and distribution of all four dataset in helix groups (HGs). All four datasets can be summarized in following points,

1. D1, The main dataset set (\# April,2014) has 1641 protein chains, which are representative structures with $85 \%$ sequence identity clusters (extracted using CD-Hit). D1 has alpha helix content varying from 1 to 50. But for purpose of our study we have considered protein structures with 3 to 10 alpha-helices (criteria used for all datasets). The total number of proteins in D1 is 977 .

2. Dataset D2 is similar to D1 but fetched from PDB on June, 2013. Total number protein chains from 3 to 10 alpha-helices, 939.

3. Dataset D3 is from Astral25, this is a domain dataset from SCOP v1.75, released on June,2009. With maximum sequence similarity between protein chains tobe $25 \%$. Total number of domains in D3 from alpha helix 3 to t10 is found to be 879 .

4. Dataset D4 is a subset of D1, with sequence identity of $25 \%$ extracted using PIECE protein chain culling server.

As mentioned in above points, from each full dataset we have selected proteins with alpha helix content minimum 3 to maximum 10 for our study. Number of proteins in each dataset with HG information has been shown in table S1. 

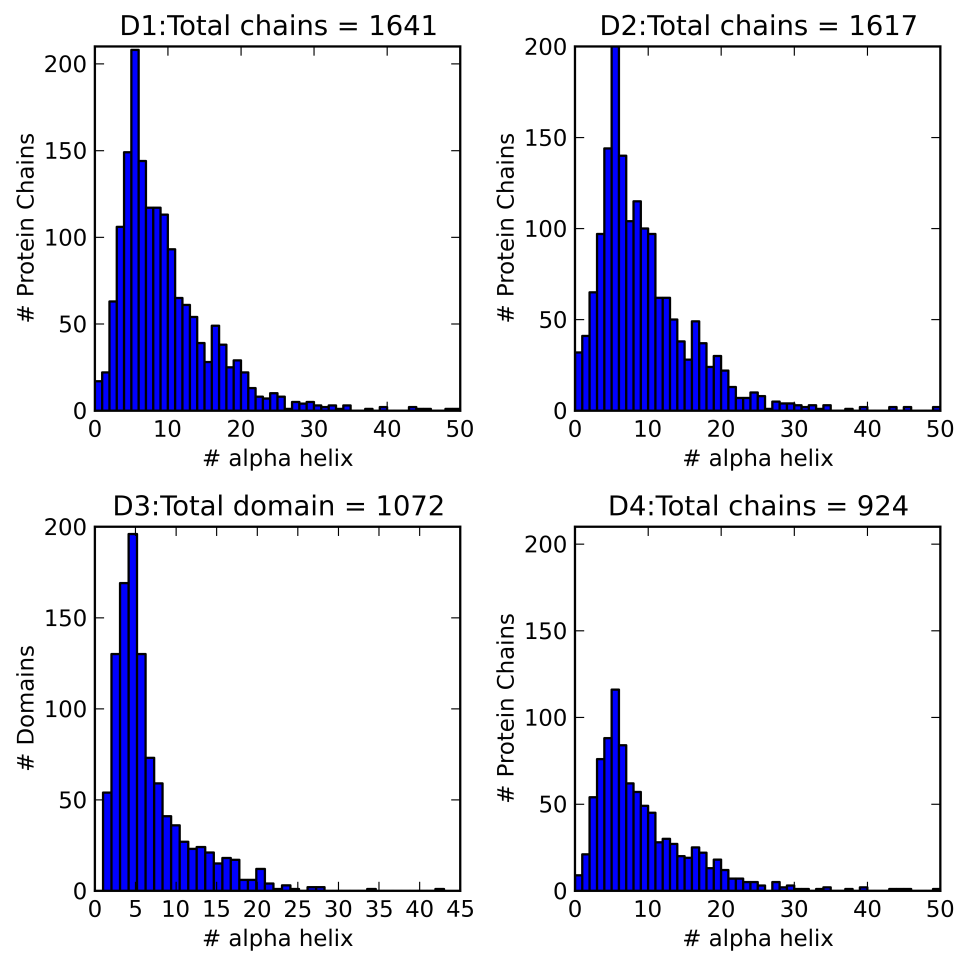

Fig.S 1: Histogram of alpha helix content in different datasets used in the current study. Datasets have been selected with similar structural criteria with different release versions (Top row D1 nad D2) as well as with altering sequence identity cut-offs (from $85 \%$ in top row to 40 and $25 \%$ bottom rows (D3 and D4 respectively)). Total number of proteins in each selection type has been mentioned in respective titles.

Table S 1: Distribution of protein chains in alpha helices

\begin{tabular}{|c|c|c|c|c|}
\hline Helix Group (HG) & D1 & D2 & D3 & D4 \\
\hline \hline 3 & 79 & 77 & 135 & 57 \\
\hline 4 & 135 & 132 & 173 & 82 \\
\hline 5 & 189 & 179 & 205 & 105 \\
\hline 6 & 137 & 133 & 136 & 80 \\
\hline 7 & 117 & 104 & 81 & 62 \\
\hline 8 & 116 & 114 & 69 & 57 \\
\hline 9 & 112 & 99 & 42 & 42 \\
\hline 10 & 92 & 96 & 38 & 48 \\
\hline Total & 977 & 934 & 879 & 533 \\
\hline
\end{tabular}




\subsection{Detail analysis of Dataset 1:}

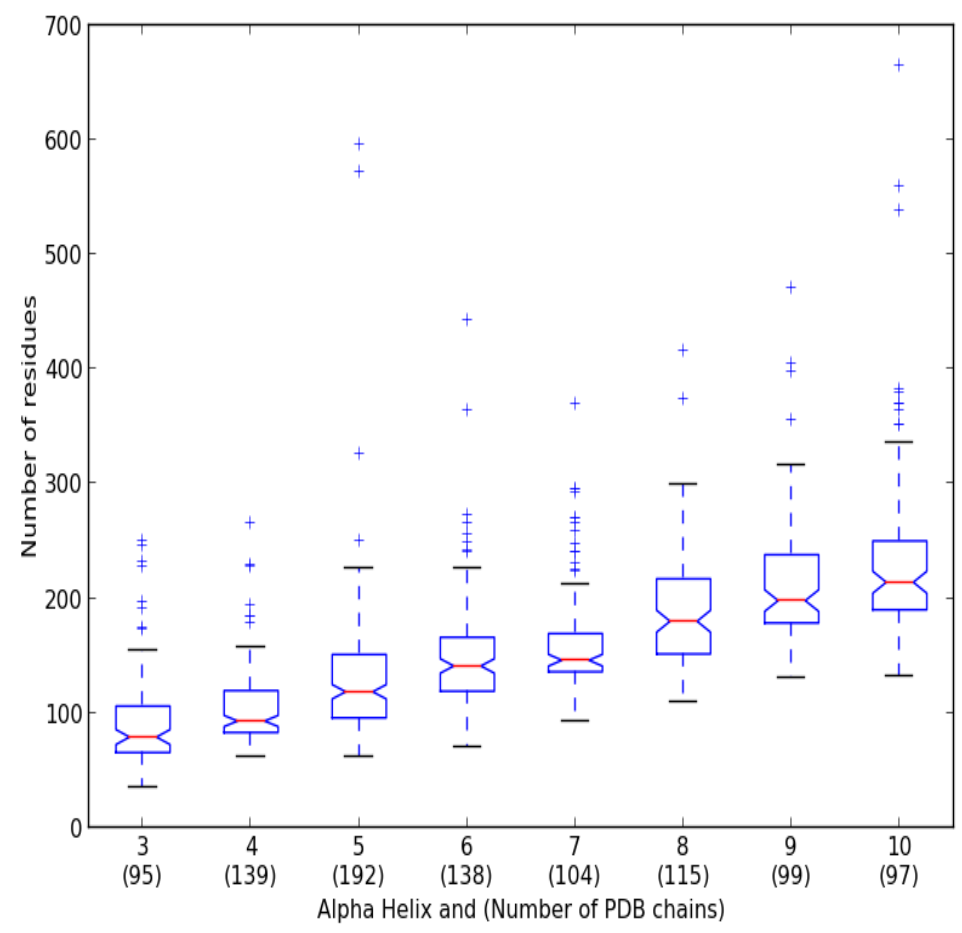

Fig.S 2: Residues in Helix Groups

Analysis of dataset protein chains in each helix group has been shown in the above notched box and whisker diagram. The $\mathrm{X}$-axis shows the protein count in different helix groups (from 3 to 10) and the Y-axis represents number of residues in a chain. The non-overlapping notches (in each case) suggests (with $95 \%$ confidence), that the average number of resides increases with increase in alpha helix count. Out layers in each case has been shown in "+" sign. The box represents inter-quartile range (IQR), with the redline showing the median of the data. The upper and lower end of the box represents 75 (Q3) and 25 (Q1) percentile of data respectively. Whiskers cover 1.5 times IQR which exceeds Q3 and which are below Q1

\subsection{Detail analysis of Dataset 3 (ASTRAL25):}

ASTRAL25, is a protein domain dataset from SCOP v1.75, released on June,2009. With maximum sequence similarity between protein chains to be $25 \%$. Total number of domains in D3 from alpha helix 3 to 10 is found to be 879 (Fig.1,Table15).

Since ASTRAL is a curated (in terms of structure and sequence) version of SCOP v1.75 and our analysis focus on helix content from 3 to 10 . D3 covers $\sim 60 \%$ structural classification data of SCOP 1.75 at lower three levels (Table2). Considering the relative orientation of interacting (or packing) helices in a protein domain, D3 has been 
Table S 2: Comparing statistics of distinct class in SCOP 1.75 (all-alpha proteins) and D3 .

\begin{tabular}{|c|c|c|c|}
\hline & Fold & Superfamily & Family \\
\hline \hline SCOP 1.75 & 284 & 507 & 871 \\
\hline D3 & 171 & 267 & 427 \\
\hline
\end{tabular}

Statistics of number of unique folds, superfamily and family in all-alpha class of proteins reported in SCOP 1.75 and that of D3 dataset. D3 dataset is a subset of ASTRAL25 (curated version of SCOP 1.75) with alpha helix content three to ten toal of 818 protein domains.

Table S 3: Analysis of D3 (ASTRAL40) dataset with respect to interacting alpha helical packing.

\begin{tabular}{|c|c|c|c|c|c|c|}
\hline HS & \multicolumn{2}{|c|}{ D3_Orthogonal ${ }^{a}$} & \multicolumn{2}{c|}{ D3_Align } \\
$b$ & \multicolumn{2}{c|}{ D3_Mixed ${ }^{c}$} \\
\hline \hline & \#domains & \#unique Patterns & \#domains & \#unique Patterns & \#domains & \#unique Patterns \\
\hline 3 & 62 & 7 & 60 & 7 & 13 & 3 \\
\hline 4 & 84 & 26 & 39 & 20 & 50 & 29 \\
\hline 5 & 54 & 33 & 69 & 51 & 82 & 70 \\
\hline 6 & 31 & 28 & 38 & 36 & 67 & 60 \\
\hline 7 & 10 & 10 & 18 & 18 & 53 & 51 \\
\hline 8 & 5 & 4 & 12 & 12 & 52 & 52 \\
\hline 9 & 2 & 2 & 7 & 7 & 33 & 33 \\
\hline 10 & 2 & 2 & 5 & 5 & 31 & 31 \\
\hline
\end{tabular}

Above table describes the distribution of protein domains (\#domains) and number of unique patterns in three subsets of D3 (all-alpha proteins from ASTRAL40) dataset. Considering the interacting helix axis orientation three different subsets has been compiled as orthogonal (a), aligned(b) and mixed(c). For each dataset cases with different number of helix set has been evaluated.

further spited into 3 different datasets. Domains with all interaction helices orientated orthogonally are grouped into D3_Orthogonal and domains with all interacting helices oriented parallel or anti-parallel fashion are clubbed in D3_Align while the rest with mixed orientations are kept in D3_Mixed dataset (Table 4). Distribution of packing angles or relative helix-axis angles of interacting helices has been illustrated in Fig. 3. Individually more packed helices are found tobe in aligned regions but after considering protein domain in total, protein domains with mixed helix orientation has maximum count (Table 3).

From table 3, it can be observed that domains with 3 and 4 helix sets are maximum in orthogonal and aligned arrangement but for higher helix sets, number domains is higher in mixed helix-axis orientation. The same can be observed for number of unique patterns. Comparing results of D3 dataset (Table S2) with three sub-dataset, distribution of number of unique patterns can be observed. 
Table S 4: Comparing prevalent patterns of D1 dataset with sub-datasets of D3

\begin{tabular}{|c|c|c|c|}
\hline \multirow{2}{*}{ HS } & \multicolumn{3}{|c|}{ \% coverage of Prevalent patterns in } \\
\cline { 2 - 4 } & D3_Orthogonal & D3_Align & D3_Mix \\
\hline \hline 3 & 66.67 & 100 & 0 \\
\hline 4 & 38.46 & 38.46 & 38.46 \\
\hline 5 & 25.64 & 23.08 & 17.95 \\
\hline 6 & 6.67 & 6.67 & 6.67 \\
\hline 7 & 8.33 & 16.67 & 16.67 \\
\hline 8 & 0 & 0 & 12.5 \\
\hline 9 & 0 & 0 & 0 \\
\hline 10 & 0 & 0 & 0 \\
\hline
\end{tabular}

Percentage coverage of prevalent pattern set as reported by study of D1 dataset (Table 3) by sub-dataset of D3. For definition of datasets see table S8 and text. Due to sparse number of representative structure in higher helix sets (7 onwards), prevalent pattern coverage sinks to zero.

Focusing on the prevalent pattern set, we have compared percentage coverage of prevalent pattern as reported in the main text (by study of dataset D1) in three sub-dataset. As number of representative structure becomes 3 fold less after splitting D3, for higher helix sets evaluation of prevalent pattern becomes statistically insignificant. From table 4 , it can be observed that minimum coverage of $\sim 13 \%$ for helix set 8 in D3_Mix whereas, maximum coverage of $100 \%$ in D3_Align for 3 helix set proteins. Cumulatively, D3 dataset shows similar sets of prevalent pattern sets across helix sets with considerable deviation of observed frequencies (xob $>=x f(+0.005)$ ).

\subsection{Distribution of protein and topology space in different datasets:}

Proteins in four dataset considered for the study (see section1), has been categorized as per number of alpha helix content. These sets or group of proteins are known as Helix Set or HS. The rational behind such classification is to comparatively study the pattern space which varies (or increase) with number of secondary structure content (see section 1.4).

Comparing the number of alpha helix content with each dataset's pattern space, it can be observed that, the numbers are not statistically significant after HS 5. Number of proteins in each HS decreases but pattern space remains expanding. By considering patterns as a module or sub-pattern in higher HS, Table 1 shows an expansion of pattern space which is $\sim 3$ times of what we observe by considering only pattern. However, from Figure 4 it can be seen the expansion of space in only fraction by considering what is possible. Growth in observed pattern space after inclusion of modules from hight HS has been similar for all four datasets, i.e. D1 : 3.4; D2: 3; D3: $\sim 2.6$ and D4: $\sim 3.4$. 


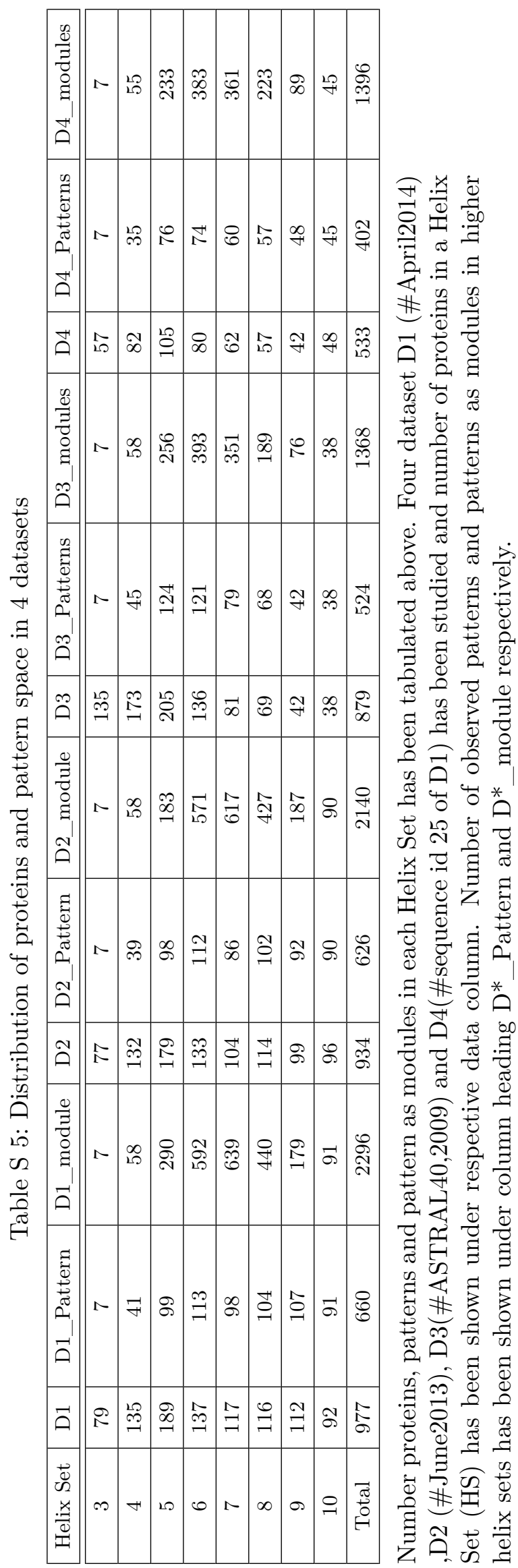




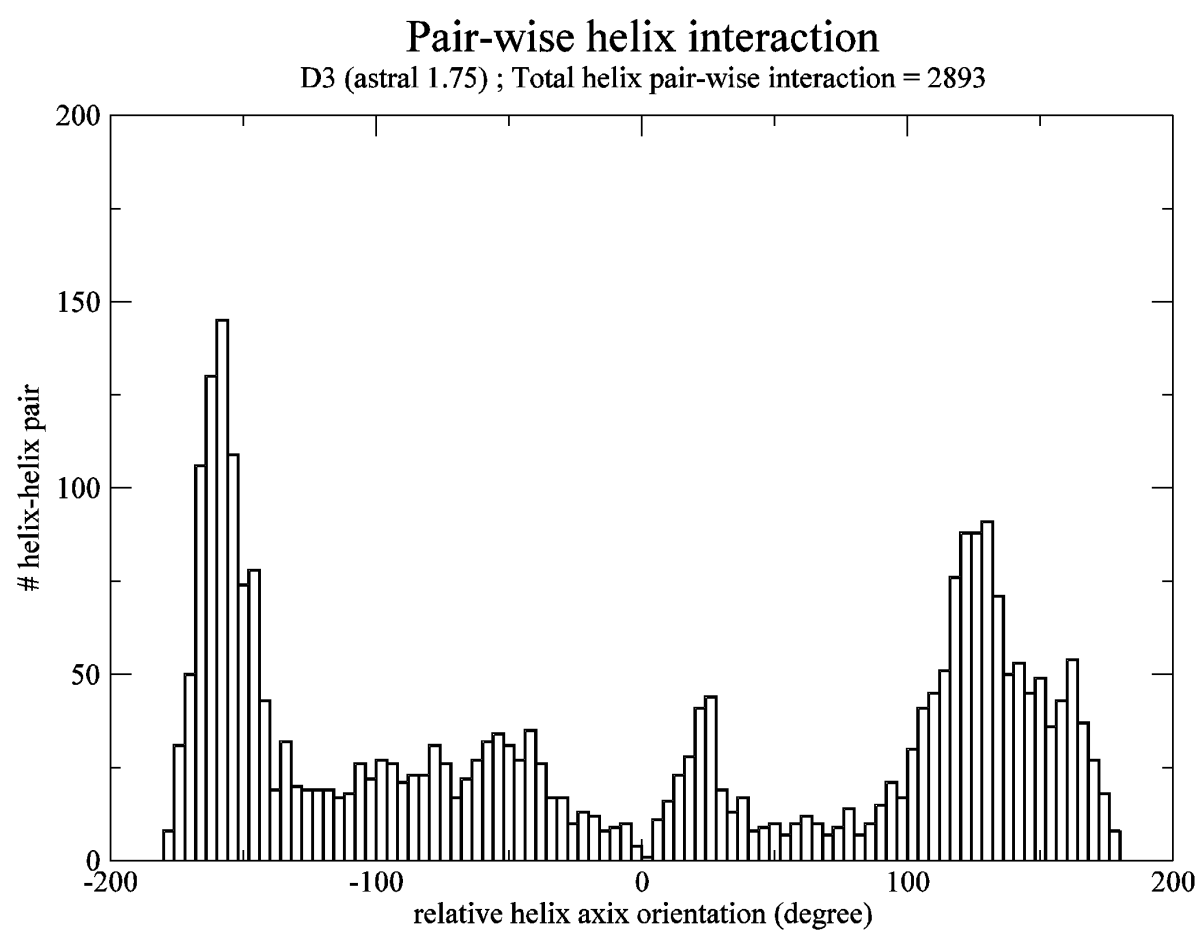

Fig.S 3: Distribution of helix interaction angles in D3 dataset.

\subsection{Expansion of Pattern space :}

Expansion of pattern space and compartive study with theoretical possibility reported in Figure 4. Total number of possible (combinatorial) is of the order of $3 * 10^{13}$ (red square). Observed pattern sum is 688 , where as inclusion of sub-patterns (or module) increases different patterns to 2661 (i.e by $286.77 \%$ ) for dataset 1 . Above figure shows these in log scale. Red line represents the vast combinatorially possible pattern space which shows to be increasing with each HS. The green line shows the observed unique patterns in log scale which seems to be increase in log scale (from $3 \mathrm{HG}$ to $5 \mathrm{HG}$ ) but don't increases thereafter. Inclusion of sub-patterns or structural modules from higher helix groups to the patterns of lower HGs shows pushing the boundaries of observed contact spaces. The expansion is substantial in natural scale as the total number of unique patterns increase by $\sim 288 \%$ (i.e. from 688 to 2661 ) . But this extensive growth in pattern space is quite low if the whole space is considered. 

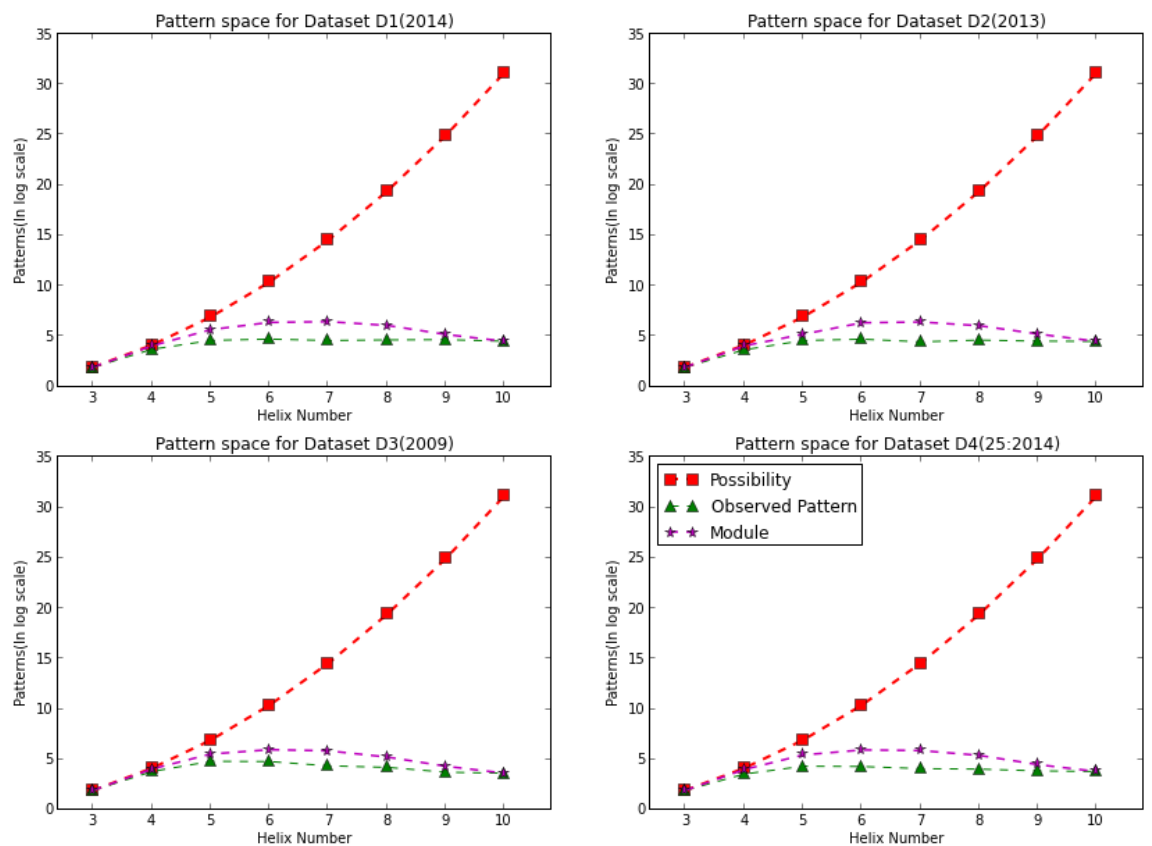

Fig.S 4: Comparing theoretically possible pattern space to observed space in 4 datasets

\section{Contact Analysis:}

The contact criteria play a defining role in our study, there-fore contact criteria with different tolerance has been tested.

Heavy atoms in protein are carbon $(\mathrm{C})$, nitrogen $(\mathrm{N})$, oxygen $(\mathrm{O})$ and sulfur $(\mathrm{S})$ and their Van der wall radius are $1.70 \mathrm{~A}, 1.52 \mathrm{~A}, 1.55 \mathrm{~A}$ and $1.80 \mathrm{~A}$ respectively. The interaction between these heavy atoms are governed by Leenard-Jones potential, which restricts atom-atom interaction to a minimum distance. Atoms can't come closure more than the sum of their van der Wall's radius and contact only possible within some threshold limits. We have analyzed contact with a contact distance threshold of $0.6 \AA$. In this section we have tested this contact distance along a range of cut offs (shown iin figure S5). 


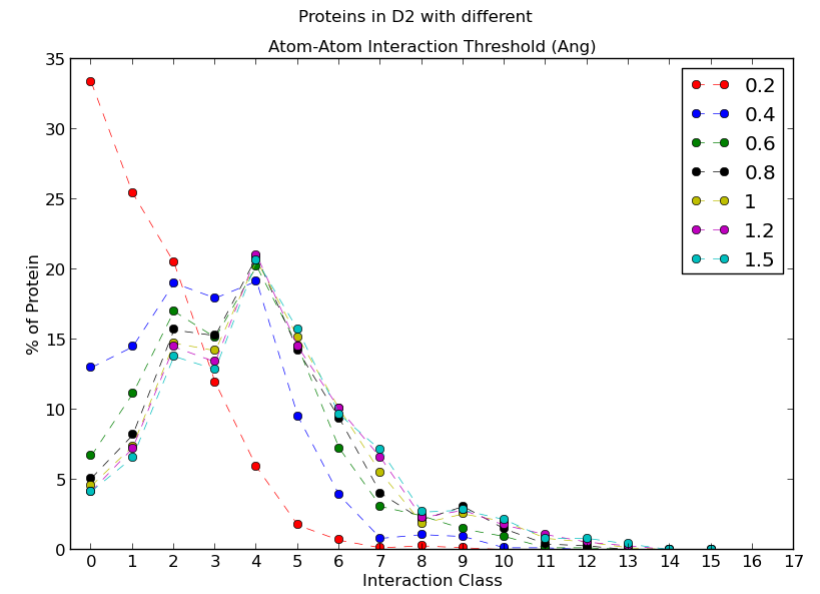

Fig.S 5: Contacting helices with change in distance cut-offs

From the figure 5 it can be observed that the percentage of protein which have no interaction is high with the low distance tolerance values. With tolerance of 0.2 (shown in red), $33.3 \%$ of protein in D1 dataset have no helix-helix packing, and this value reduces to $12.9 \%$ in $0.4 \mathrm{~A}$ and $6.6 \%$ in $0.6 \mathrm{~A}$ and as we increase the tolerance this value doesn't change vary much (from $5.08 \%$ in $0.8 \mathrm{~A}$ to $4.15 \%$ in $1.5 \mathrm{~A}$ ). Percentage of proteins with one helix-helix packing pair also reduces from $25.4 \%$ in $0.2 \mathrm{~A}$ tolerance to $11.1 \%$ in $0.6 \mathrm{~A}$ and $8.16 \%$ in $0.8 \mathrm{~A}$ but remains $~ 7 \%$ as further increase in tolerance. There is minor change in percentage of protein in 2 interaction class i.e. proteins which has two helix-helix packing pair as $20.4 \%$ in $0.2 \mathrm{~A}$ to $17 \%$ in $0.6 \mathrm{~A}$ and $13.7 \%$ in $1.5 \mathrm{~A}$ as shown in figure 5 . In interaction class 3,4 and 5 there is increasing number of protein with increase in contact tolerance.

\section{Statistical significance for distribution}

We have tested hypothesis (null hypothesis), that all the contacts have same proportion of protein in a HG. As our data is multinomial (more than one category), we perform test using chi-square distribution and the test performed is goodness-of-fit. In goodness-of-fit , we test the null hypothesis, that the observed frequencies for an experiment follow a certain pattern or theoretical distribution. The statistical test has been done on $1 \%$ level of significance, with null hypothesis as "numbers of protein are equally distributed among various contacts in a HG". If the test result $(\chi 2)$ is less than or equal to the critical value (is the value from the chi-square distribution table for a particular degree of freedom at $1 \%$ significance level), test will support the null hypothesis. This indicates that at $1 \%$ level of significance, the proportion of pdb structure in each contact is same. Otherwise, the proportions are not equally probable and the probability of finding distribution like observed by chance is less than 0.01 (Table S6,7). 
Table S 7: Statistical significance Test for occurance of pattern as modules in Dataset 1

\begin{tabular}{|c|c|c|c|c|c|c|}
\hline HS & Rep. Str. & Pattern Count & Expected Val & chi_Square & DF & Is P-val $<0.001$ \\
\hline \hline 3 & 9177 & 7 & 1311 & 4053.814 & 6 & yes \\
\hline 4 & 7892 & 58 & 136.069 & 17146.773 & 57 & yes \\
\hline 5 & 5284 & 290 & 18.221 & 20850.357 & 289 & yes \\
\hline 6 & 3167 & 592 & 5.35 & 7745.652 & 591 & yes \\
\hline 7 & 1698 & 639 & 2.657 & 1615.919 & 638 & yes \\
\hline 8 & 719 & 440 & 1.634 & 206.897 & 439 & NO \\
\hline 9 & 183 & 179 & 1.022 & 5.781 & 178 & NO \\
\hline
\end{tabular}

Table S 6: Statistical significance test (Chi-square test) for classifying proteins into pattern space in each Helix Set (HS)

\begin{tabular}{|c|c|c|c|c|c|c|}
\hline Helix Set & \# of Proteins & $\begin{array}{c}\text { Observed } \\
\text { Patterns }\end{array}$ & Expected Values & $\chi^{2}$ & $d f$ & Is p-value $<0.001$ \\
\hline \hline 3 & 79 & 7 & 11.286 & 26.886 & 6 & yes \\
\hline 4 & 135 & 41 & 3.293 & 87.007 & 40 & yes \\
\hline 5 & 189 & 99 & 1.909 & 147.81 & 98 & yes \\
\hline 6 & 137 & 113 & 1.212 & 32.088 & 112 & no \\
\hline 7 & 117 & 98 & 1.194 & 49.684 & 97 & no \\
\hline 8 & 116 & 104 & 1.115 & 22.069 & 103 & no \\
\hline 9 & 112 & 107 & 1.047 & 8.375 & 106 & no \\
\hline 10 & 92 & 91 & 1.011 & 0.978 & 90 & no \\
\hline
\end{tabular}

Statistical significance of classification of proteins in contact classes of a helix group has been shown in above table. Test has been done for significance of classification greater than $.5 \%$ ( $\mathrm{p}$-value $>0.005$ ). This means that the chance of achieving this classification by random is less than $0.1 \%$. Analyzing the Chi-square value and degree of freedom (df) from Chi-square distribution table decision has been taken and shown in last column.

\section{Structural Descriptor Analysis:}

We have analyzed different physico-chemical and structural properties in different contact of a HG (Fig. 3 main text). Four structural descriptors are discussed in following sections detail in figure6

- a. Relative contact order ( $\mathrm{rCO})$ : quantifies the presence of local contacts (i.e. atom contact within $6 \AA$ ) in a protein. This descriptors value will be less for proteins with high local contacts (eg. Helical proteins) and relatively more for protein with non-local contacts (beta proteins). As all proteins in our dataset has high presence of alpha helices, from figure 2 we can observe relatively comparable scores (range of 0.06 to 0.10 ), which is found to be within the standard deviation of relative 
contact order reported earlier rCO also known to be inversely related to folding kinetics.

- b. Normalized radius of gyration $(\mathrm{RgN})$ : Radius of gyration of a protein has been used as a measure of protein compactness. Normalized measure of compactness has been proposed by Ivankov et al. To analyze the difference in compactness profiles we have calculated average normalized radius of gyration $(\mathrm{RgN})$ of proteins in a contact for each HGs. Our results indicate that $\mathrm{RgN}$ values within a range of 1.2 to 1.5. Present study shows that, alpha-proteins are less compact then a sphere of equal volume.

- c. Percentage loss in helix accessibility (LHSA): Solvent accessibility loss of a helix for being in a particular contact pattern has been investigated. We have calculated the loss by comparing solvent accessibility of a helix isolated from protein with accessibility of same helix in the protein. For almost every helix group we found the accessibility loss is approximately $40 \%$, which is in accordance to early reported loss in packed helices. d. Helix hydrophobicity (Helix Hyd.): Analyzing hydrophobicity of residues in helices we found that across different contact class and helix-groups, average hydrophobicity score is $>1$.

- e. We have explored various structural features of proteins by analyzing structural descriptors. These relations for $\mathrm{rCo}$ and $\mathrm{RgN}$ have been established for small globular proteins (amino acid residue count $>100$ ), which is different in this case. Form all descriptor analysis, it can be comprehended that by structural features there is minor difference between different contacts with the observed variance. 

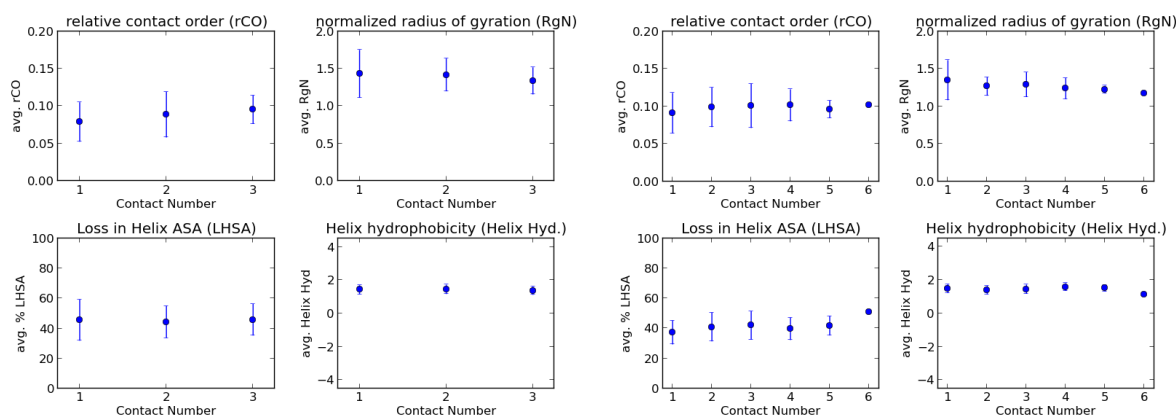

(a) $3 \mathrm{HG}$

(b) 4 HG
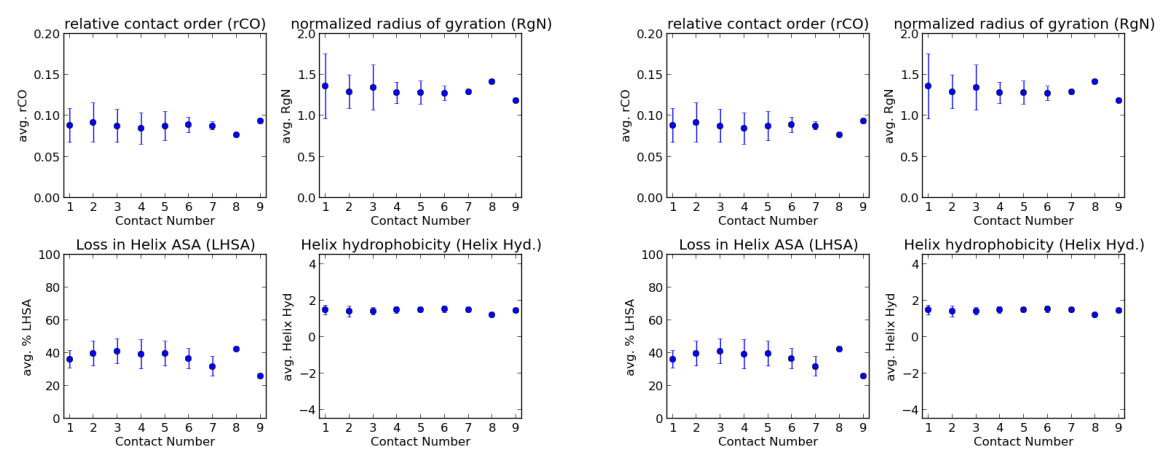

(c) $5 \mathrm{HG}$

(d) $6 \mathrm{HG}$
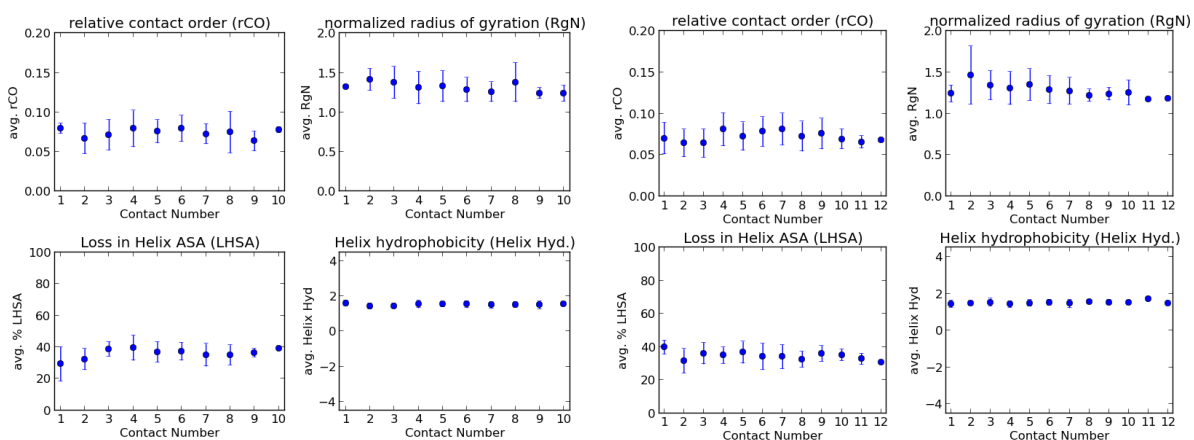

(e) $7 \mathrm{HG}$

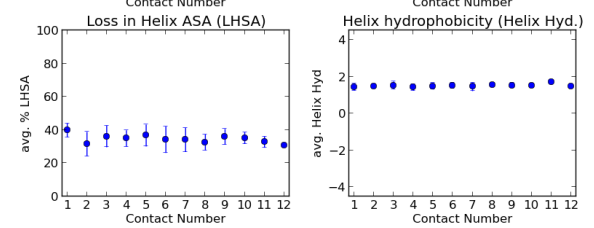

(f) $8 \mathrm{HG}$

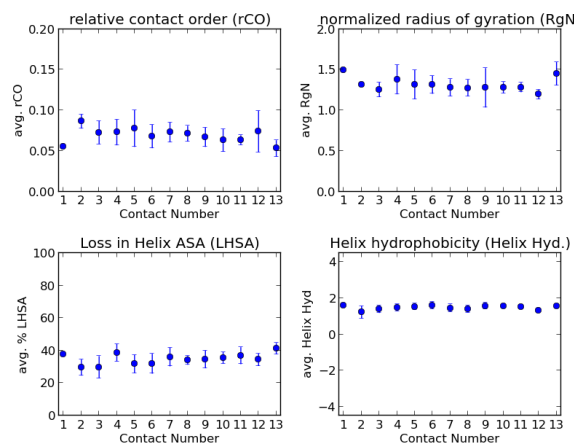

(g) $9 \mathrm{HG}$

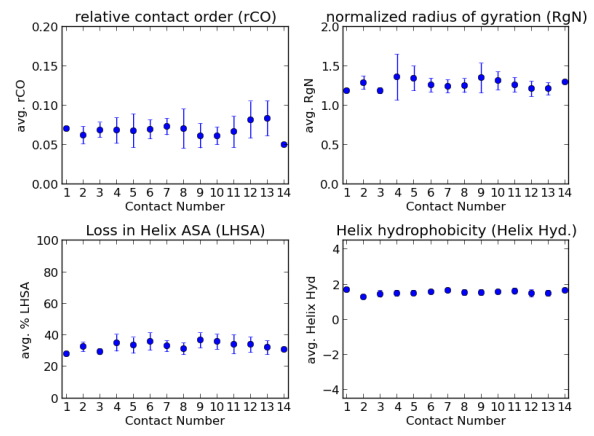

(h) $10 \mathrm{HG}$

Fig.S 6: Structural descriptors in Dataset 1 
Table S 8: Detail functional annotation by SUPERFAMILY of proteins in D1 dataset

\section{Function Analysis}

Table S4 shows the functional annotation of proteins in our dataset of 979 proteins. Superfamily annotates proteins in 7 general functions (sl no 1-7) and 50 detail function. For functional annotation we have used latest functional annotation file by SUPERFAMILY (scop.annotation.1.73) . ( $\mathrm{m} / \mathrm{tr}$ - metabolism and transport) . Complete distribution of functional classes across helix group has been given in figure 7 .

Table 4: Presence of functional groups in D1 dataset

\begin{tabular}{|c|c|c|c|}
\hline Sl.No & $\begin{array}{l}\text { General Function } \\
\text { (total protein) }\end{array}$ & Detail Function & $\begin{array}{c}\text { Number of } \\
\text { Proteins }\end{array}$ \\
\hline \multirow{4}{*}{1} & \multirow{4}{*}{$\begin{array}{l}\text { Extra-cellular } \\
\text { processes }(50)\end{array}$} & Immune response & 27 \\
\hline & & Toxins/defense & 14 \\
\hline & & Cell adhesion & 8 \\
\hline & & Blood clotting & 1 \\
\hline \multirow{4}{*}{2} & \multirow{4}{*}{ General (96) } & Protein interaction & 28 \\
\hline & & General & 52 \\
\hline & & Small molecule binding & 15 \\
\hline & & Ligand binding & 1 \\
\hline & & & \\
\hline \multirow{5}{*}{3} & \multirow{5}{*}{ Information (80) } & Translation & 14 \\
\hline & & DNA replication/repair & 27 \\
\hline & & RNA processing & 5 \\
\hline & & Transcription & 33 \\
\hline & & Chromatin structure & 1 \\
\hline \multirow{8}{*}{4} & \multirow{8}{*}{$\begin{array}{l}\text { Intra-cellular processes } \\
(121)\end{array}$} & Transport & 17 \\
\hline & & Cell cycle & 8 \\
\hline & & Phospholipid $\mathrm{m} / \mathrm{tr}$ & 25 \\
\hline & & Protein modification & 23 \\
\hline & & Trafficking/secretion & 4 \\
\hline & & Ion $\mathrm{m} / \mathrm{tr}$ & 36 \\
\hline & & Proteases & 6 \\
\hline & & Cell motility & 2 \\
\hline \multirow[t]{3}{*}{$(296)^{\prime \prime}$} & & Lipid $\mathrm{m} / \mathrm{tr}$ & 12 \\
\hline & & Amino acids $\mathrm{m} / \mathrm{tr}$ & 6 \\
\hline & & Redox & 78 \\
\hline Sl.No & $\begin{array}{l}\text { General Function } \\
\text { (total protein) }\end{array}$ & Detail Function & $\begin{array}{c}\text { Number of } \\
\text { Proteins }\end{array}$ \\
\hline
\end{tabular}




\begin{tabular}{|c|c|c|c|}
\hline \multirow[t]{12}{*}{ Sl.No } & $\begin{array}{c}\text { General Function } \\
\text { (total protein) }\end{array}$ & Detail Function & $\begin{array}{c}\text { Number of } \\
\text { Proteins }\end{array}$ \\
\hline & & Photosynthesis & 5 \\
\hline & & Energy & 75 \\
\hline & & Other enzymes & 45 \\
\hline & & Polysaccharide $\mathrm{m} / \mathrm{tr}$ & 1 \\
\hline & & E- transfer & 40 \\
\hline & & Nitrogen $\mathrm{m} / \mathrm{tr}$ & 2 \\
\hline & & Carbohydrate $\mathrm{m} / \mathrm{tr}$ & 3 \\
\hline & & Transferases & 10 \\
\hline & & Nucleotide $\mathrm{m} / \mathrm{tr}$ & 9 \\
\hline & & Secondary metabolism & 4 \\
\hline & & Coenzyme $\mathrm{m} / \mathrm{tr}$ & 6 \\
\hline \multirow[t]{2}{*}{6} & Other & Viral proteins & 20 \\
\hline & & Unknown function & 32 \\
\hline & & & \\
\hline \multirow{6}{*}{7} & \multirow{6}{*}{ Regulation (211) } & DNA-binding & 138 \\
\hline & & RNA binding & 3 \\
\hline & & Signal transduction & \\
\hline & & Receptor activity & 7 \\
\hline & & Other regulatory function & 11 \\
\hline & & Kinases/phosphatases & 5 \\
\hline Sl.No & $\begin{array}{c}\text { General Function } \\
\text { (total protein) }\end{array}$ & Detail Function & $\begin{array}{c}\text { Number of } \\
\text { Proteins }\end{array}$ \\
\hline
\end{tabular}



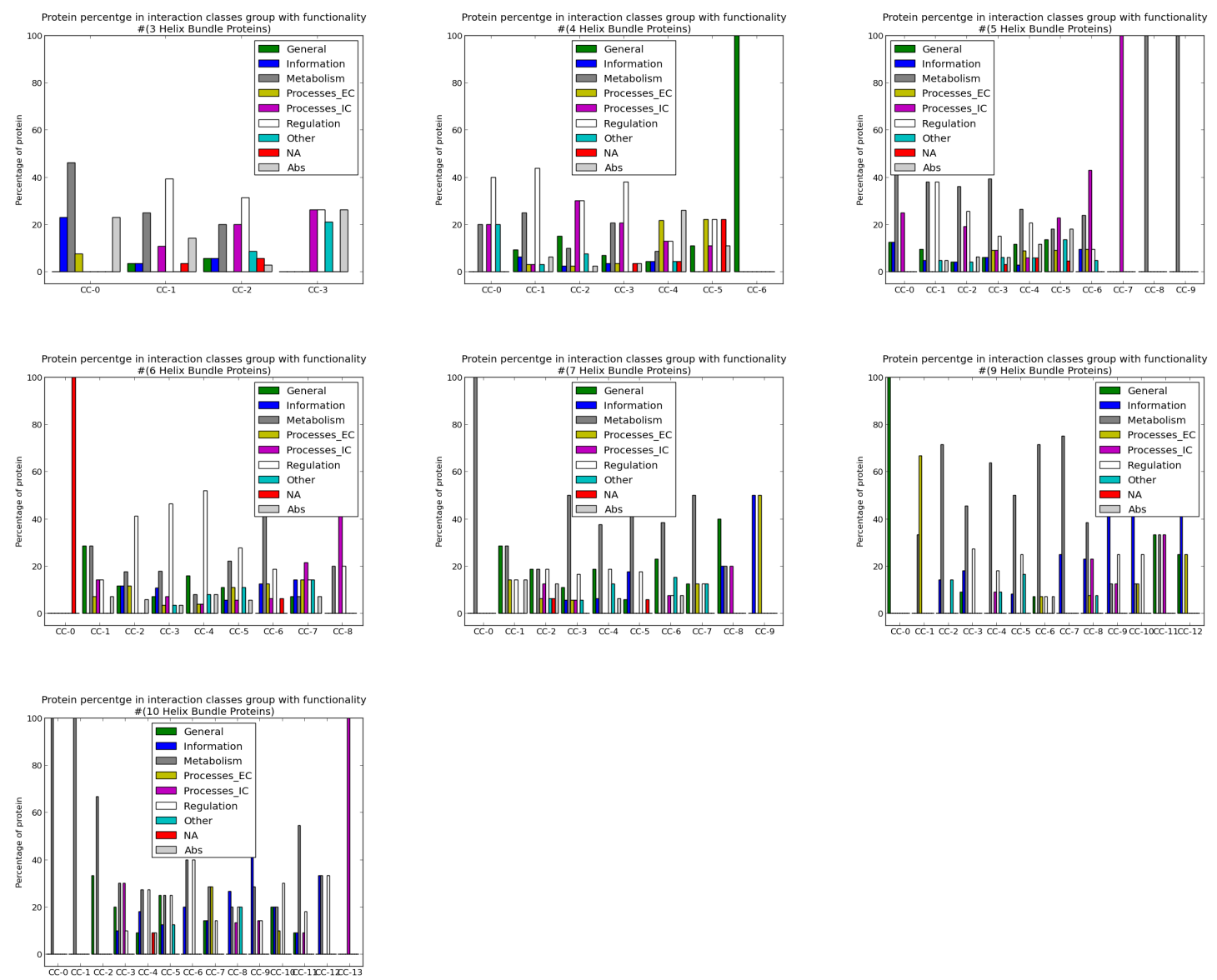

Fig.S 7: Distribution of functional classes ("general" across helix groups)

\section{Enzymes in dataset}

Out of 952 proteins, our dataset ${ }^{1}$ has 175 protein chains with enzymatic activity. Presence of nearly $82 \%$ of non-enzyme is arguably due to the choice of protein class, which is agreeable to the earlier studies [1]. Analyzing enzymes in dataset, it was observed that oxidoreductase (Oxi), transferase (Tras) and hydrolase (Hyd) are present in nearly equal proportion, which covers most of the dataset $(\sim 87.4 \%)$. Lyase, Isomerase and Ligase have compartively lower representative $(\sim 12 \%)$ (Main text Table 5 ).

\subsection{Distribution of ECs in Helix Groups:}

Distribution of enzymes in different EC classes has been described in table (main 5). With the increase in alpha helix content, percentage of enzymes in dataset decreases

\footnotetext{
${ }^{1}$ of alpha helices
} 
from $19.4 \%$ in 4 helix group proteins to $8.65 \%$ in case of 7 helix group proteins, which has minimum ratio of enzyme - non-enzymes. But percentage enzymes in dataset are observed to be 3 times more in case of 8,9 and 10 helix group proteins i.e. $28.69 \%, 28.28 \%$ and $26.8 \%$ respectively. This trend can has been illustrated in Figure 8 .

Fig.S 8: Percentage of enzymes in Helix groups of alpha dataset

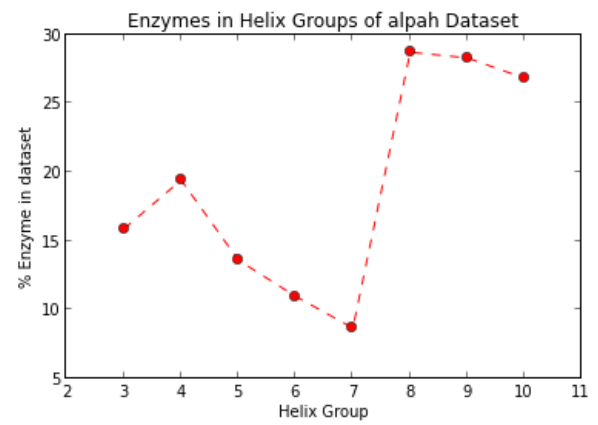

Above figure shows helix group wise percentage of enzymes in alpha dataset. Total number of enzymes in dataset is 175 , whereas there are 952 proteins in the dataset.

Six enzyme classes are present in different proportion in each helix group as can be observed in table. The lower HGs (i.e. 3,4 and 5) has dominantly higher presence of hydrolases (ec: 3.---.-), whereas higher HGs (8,9 and 10) have higher oxidoreductase (ec: 1.-.-.-) and transferase ( ec: 2.-.-.-). To check, how these numbers are statistically significant we have performed chi-square test, which is appropriate for our case of multiple population [2]. From statistical significance test, being p-value minimum of 0.005 (for HG 4,5,8,9 and 10) and maximum less than 0.1 (for HG 3 and 7), it can be safely stated that the distribution is not by random event.

\subsection{Comparing all enzymes reaction in dataset:}

Comparing enzyme reaction gives advantage of understanding protein function by its biochemical mechanism. Type of reaction and its mechanism could be used to explore the chemical diversity based on type of bond change in mechanism, change in local environment of catalytic site or change in type of structure act as substrate.

To analyze the diversity of biochemical reactions among enzymes of our dataset we have compared all enzymes of our dataset who's reaction is well resolved and balanced. The total number of proteins with assigned e.c. number is 175 out of 952 total proteins. Total number of different e.c. number are 88. Among 88 different enzymes, there are 12 e.cs which do not have resolved e.c number, hence detail of reaction was not known. Out of 76 known enzymes, there are only 24 e.cs whose balanced reaction was found from Rhea $[3,4]$. Detail of reaction description by kegg and corresponding igs can be viewed from (data_module-sheet:ReactionDetail.xls).

Comparing enzyme reaction in 24 balanced reactions (64 proteins) has been performed in EC-Blast as described in the 'Method' section. Similarity between enzymes are com- 
Table S 10: Distribution of protins from D1 dataset in different Enzyme Classes

\begin{tabular}{|c|c|c|c|c|c|c|c|c|c|c|}
\hline HG & Non-Enz & Enz & Oxi & Trans & Hyd & Ly & Iso & Lig & Chi $^{\wedge} 2$ & P-value \\
\hline \hline 3 & 69 & 13 & 3 & 2 & 5 & 1 & 2 & 0 & 68 & $<0.1$ \\
\hline 4 & 108 & 26 & 2 & 6 & 14 & 1 & 0 & 3 & 30.76 & $<0.005$ \\
\hline 5 & 159 & 25 & 7 & 4 & 11 & 3 & 0 & 0 & 21.8 & $<0.005$ \\
\hline 6 & 122 & 15 & 3 & 6 & 5 & 1 & 0 & 0 & 13.4 & $<0.01$ \\
\hline 7 & 95 & 9 & 2 & 2 & 2 & 2 & 1 & 0 & 2.3 & $<0.1$ \\
\hline 8 & 82 & 33 & 11 & 14 & 5 & 0 & 2 & 1 & 30.09 & $<0.005$ \\
\hline 9 & 71 & 28 & 10 & 12 & 5 & 0 & 1 & 0 & 29.85 & $<0.005$ \\
\hline 10 & 71 & 26 & 11 & 8 & 3 & 2 & 1 & 1 & 20.15 & $<0.005$ \\
\hline Total & 777 & 175 & 49 & 54 & 50 & 10 & 7 & 5 & & \\
\hline
\end{tabular}

Distribution of enzymes present in dataset D1 has been shown in above table. Each row shows helix set (HS) wise distribution of proteins in six enzyme class i.e. Oxidoreductase (Oxi), Transferase (Trans), Hydrolase (Hyd), Lyase (Ly), Isomerase (Iso) and Ligase (Lig). Protein chains which don't have any assigned E.C numbers are represented as Non-enz. Statistical significance of distribution of E.Cs in each HS has been tested with chi-square $\left(\chi^{2}\right)$ test and their respective p-values have been reported.

prehend by comparing generated fingerprints on bond change, reaction center and reaction structure similarity, quantified in tanimoto coefficient in the range of 0 to 1 . Similarity score of 0 means the fingerprints of comparing features are different or least similar, whereas score of 1 represent high similarity. Figure 9, shows range and distribution of similarity score in all three features. Mean of all three similarity matrix is 0.2 , shows the diversity in reaction types. Bond changes has higher similarity scores in compare to other two. Outliers in Bond change, with higher similarity score is due to the presence of similar sub-sub classes (viz. 1.2.x.x (2), 3.6.x.x (3),3.1.x.x(2), 4.2.-.- (3)). Reaction center being with in score of 0.5 represents high local chemical environment variance. On the other hand due to presence of common substrate (ATP , NADP), structure similarity data is more sparsely populated. 
Fig.S 9: Comparing similarity of all-alpha enzymes

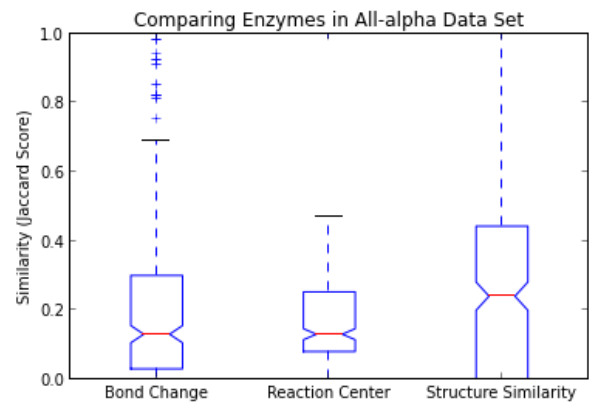

Above figure shows the range and distribution of three similarity scores of enzymes in allalpha dataset. The box represents inter-quartile range (IQR), with the redline showing the median of the data. The upper and lower end of the box represents 75 (Q3) and 25 (Q1) percentile of data respectively. Whiskers cover 1.5 times IQR which exceeds Q3 and which are below Q1. Outliers are shown in ' + ' sign. The similarity scores are in the range 0 to 1 , where 0 represents dissimilar and henceforth. Similarity scores are calculated in tanimoto coefficient by comparing finger prints of bond change, reaction center and structure similarity.

\subsection{All-vs-all enzyme reaction mechanism comparison}

Detail all-vs-all comparison of enzyme reaction has been performed with EC-BLAST and analyzed. Total number of ecs for this study is 24 whose reaction are balanced and rxn files are available. Figure 10 illustrates the similarity scores as heat map scatter plot. The plot formed a $24 * 24$ diagonal matrix, whose elements contains similarity scores in color grid. Dense the color more is the similarity and vice-versa. 
Fig.S 10: All-vs-all enzyme reaction comparison

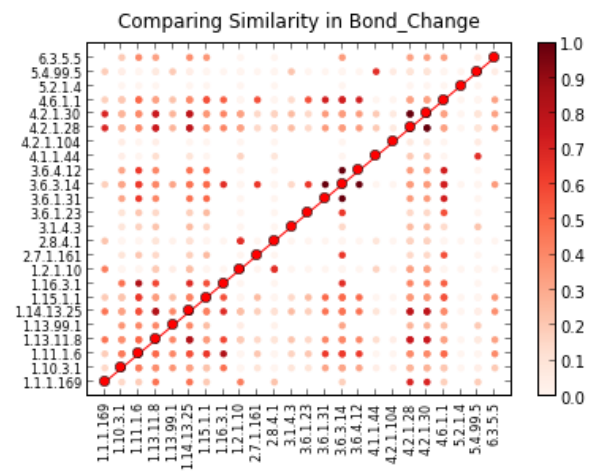

(a) Bond Center Similarity

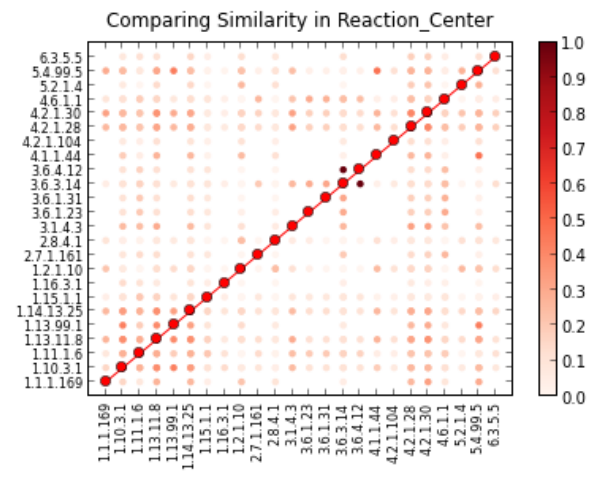

(b) Reaction Center Similarity

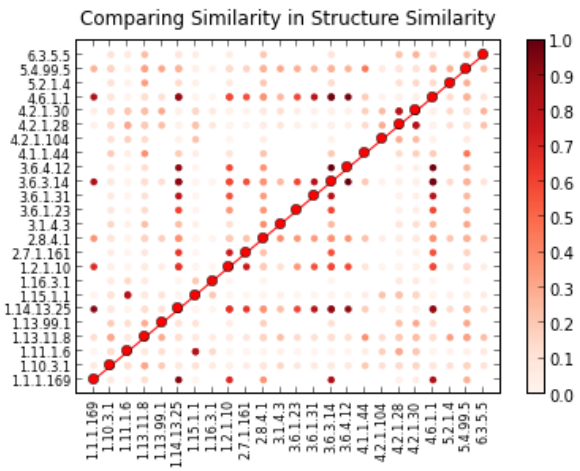

(c) Structure similarity

All-vs-all comparison of ECs in alpah dataset. Total balanced reaction in dataset is of 24 e.cs, whereas total enzymes is that of 77 excluding non-resolved ecs. Heat map shows the value of similarity score at each ec-ec comparison.

As shown in figure 8, bond changes of enzymes with same sub-classes are highly similar. Among all three descriptors reaction center are observed to have more diverse change. This is shown by high color density points. Again due to presence of similar substrates like ATP and NADP (ref). enzymes with similar substrates are found to have higher similarity scores $(\sim 0.8)$.

\section{References}

[1] H Hegyi and M Gerstein. The relationship between protein structure and function: a comprehensive survey with application to the yeast genome. Journal of molecular biology, 288(1):147-64, April 1999.

[2] P. Mann. Introduction to Statistics. John Wiley \& Sons Inc, 1999. 
[3] Abdullah Kahraman, Richard J Morris, Roman a Laskowski, and Janet M Thornton. Shape variation in protein binding pockets and their ligands. Journal of molecular biology, 368(1):283-301, April 2007.

[4] Syed Asad Rahman, Sergio Martinez Cuesta, Nicholas Furnham, Gemma L Holliday, and Janet M Thornton. EC-BLAST: a tool to automatically search and compare enzyme reactions. Nature methods, (January):1-7, January 2014. 